\title{
Innovation and Prospect of Sports Industry Reform in the Era of Internet -Taking Sports APP Operation as an Example
}

\author{
Yanjing Pan ${ }^{1, a}$, Xue Ding ${ }^{2, \star}$, Changhong $\mathrm{Xu}^{1, \mathrm{~b}}$ and Honghong Yang ${ }^{3, \mathrm{c}}$ \\ ${ }^{1}$ Institute of Physical Education, Yunnan Normal University, Kunming 650500, China \\ ${ }^{2}$ Office of Admissions, Yunnan Normal University, Kunming 650500, China \\ ${ }^{3}$ Institute of Art, Yunnan Normal University, Kunming 650500, China \\ a704019088@qq.com, 'b1531419886@qq.com, 'bbyhyanghonghong@163.com \\ *Corresponding Author: Xue Ding
}

Keywords: Sports Industry; Innovation; Development; Reform.

\begin{abstract}
With the prosperity of the global economy and the rapid development of Internet era, the potential economic value of sports industry is becoming more and more apparent. The integration of sports industry and Internet will become the new trend of China's economic development.
\end{abstract}

\section{The development of sports industry in the Internet Era}

\subsection{Sports industry development background}

Compared with China's sports industry, the sports industry in developed countries has been relatively mature and perfect. The core of foreign sports industry is fitness, leisure and competition. Integration, the formation of a huge market system size. Compared with the developed countries, China's sports industry started late, limited size. In 2015 the proportion of the national sports industry GDP (Table 1) as a comparison, the proportion of China's sports industry is low. In recent years, although China's sports industry in a substantial increase, but there are still the following questions: First, the overall production volume is insufficient, the production structure is not rigorous, the production structure is not strict, The development of the main market is not sufficient; Second, the development of sports resources is not sufficient, a large number of the lack of sports venues, sports copyright, the market allocation of resources in the decisive role has not been fully developed; Third, the lack of effective measures to stimulate the development of sports industry, The enthusiasm is not effectively mobilized and protected; Fourth, the government to promote the sports industry work system is imperfect, not yet complete with the continuity of the sector linkage, sports industry, fitness and leisure as an important part of the daily life of the masses, the market potential to be further excavated .

Table 1. The proportion of the national sports industry GDP by 2015

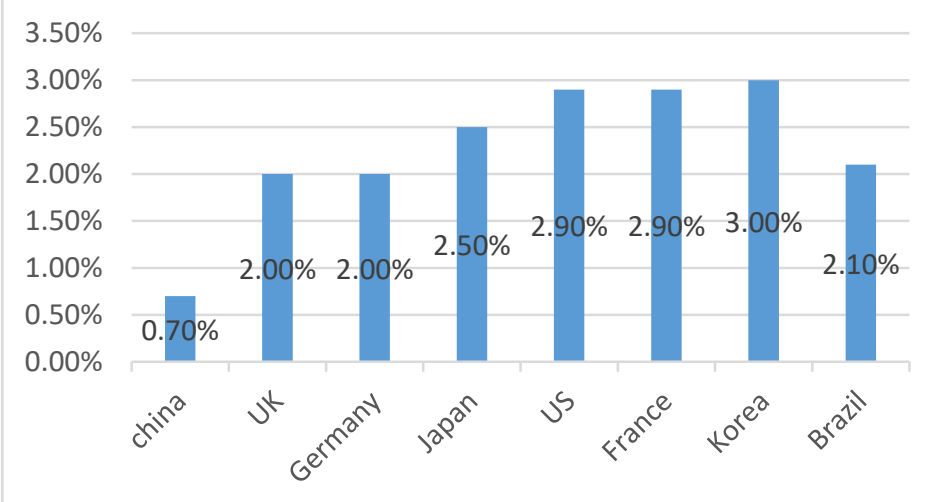

\subsection{Analysis of the Development Advantages of Sports Industry in the Internet Age}

Technical aspects of analysis: broadband business tends to improve. With the development of data communications and multimedia services, China has entered the era of 4G, 4G has a high data transmission speed, communication network developed to the sports media transmission channels 
and ways to provide the perfect conditions and opportunities. The integration of network technologies enables users to enjoy a variety of services. The convergence of applications and content experiences leads to a complex and diversified value chain. Terminal technology enables mobile phones to support more functions, including credit cards, multimedia devices, personal computers and game consoles. And other capabilities. Internet-derived social software, such as paste it, weibo, forums, etc., have set up the sports sector, sports and social information for sports to provide a platform.

Participation level analysis: the promotion of national fitness awareness. In today's society, people gradually meet the needs of the economy, but also aware of the importance of good health. More and more people are willing to spend time and energy to participate in various sports activities. In recent years, the proportion of expenditure on sports consumption gradually increased, the public sports facilities for the national fitness awareness has played a pad to improve the role. By 2010, the "overall fitness program" provides the goal has been completed, in order to further develop the national fitness career, the government developed and implemented the national fitness program. Extensive national fitness activities.

Policy level analysis: the national government to support the development of sports industry. The government has increased the financial investment at all levels of public health, encourage the community to establish a comprehensive fitness campaign. The relevant departments of the State Council to develop real-time public sports facilities, given the policy and financial great support. "The State Council on accelerating the development of sports industry to promote sports consumption of a number of opinions," the introduction of the full expression of the party and the state structure of the people's livelihood, accelerate the development of the service industry, vigorously develop the sports industry's attention, to further enhance the sports industry in the national economic development In the position.

\section{To sports APP operation as an example of specific analysis}

2016, is recognized as the sports year, the European Cup, the Olympic Games and other world-class events, set off a wave after another wave of sports circles. However, it is also the Chinese Internet has made leaps and bounds development year. From 2015 to start a substantial personalized news client growth, extending to overseas clients, and personalized recommendation technology in more areas play a role in the use; sports on the network operation, mainly used in the mobile phone APP, micro letter public Account number and so on. And as the hottest sport of APP, there are mainly four types.

First, to livescore as a representative of the simple sports data class app, the user is used to view the game score, the app is to update the data information, score information, points to the user rankings and push. This is a relatively simple sports app, user-friendly simple, widely distributed in the world of users.

Second, is a comprehensive content class sports app, contains a large number of sports data, video, depth of the article, live events, etc., each national sports category downloads are basically the largest integrated class app, but the internationalization and localization More difficult. Comprehensive sports app also has a special form: for individual events and the introduction of their names are often directly with the name of the game, such as the European Cup, Indiana Super League, etc., during the game has a large number of downloads, Mainly used during the event. China's comprehensive sports App mainly to music as sports, Tencent Sports, Sina sports integrated AAP-based.

Third, is the live app, to live real-time events? Users can use the APP without the constraints of the venue to watch the real-time game, the APP has a review function, the user can and users around the same time communicate. From the overall trend point of view, and now the major sports industry in the development of sports APP. The sports industry under the Internet has a good development prospect.

Fourth, is the fitness to lose weight APP, this kind of APP to diet movement recording and the analysis primarily? People pay more and more attention to health problems, the user through the record of running data, testing physical health, and then further make a reasonable fitness program. 
On the other hand, in the network platform to establish the sports community to share fitness experience, sports data, interactive cooperation, increase APP activity and downloads.

According to the active penetration of sports APP users in the Chinese market (Table 2), sports APP, Tencent sports and other integrated sports APP accounts for a large proportion because of its convenient operation, large amount of information, content Rich, so a high degree of activity, more by the user's welcome. Has good prospects for development. (Figure: active penetration = app number of active users / active users in China market)

Table 2. Sports APP active penetration

\begin{tabular}{|c|c|}
\hline Sports app & Active permeability \\
\hline Lesports & $0.1625 \%$ \\
\hline Tencent sports & $0.1343 \%$ \\
\hline Dongqiu sports & $0.093 \%$ \\
\hline Hupu sports & $0.0647 \%$ \\
\hline Zhangyu TV & $0.0324 \%$ \\
\hline Sina sports & $0.0319 \%$ \\
\hline A8 sports live & $0.0221 \%$ \\
\hline Storm sports & $0.0145 \%$ \\
\hline
\end{tabular}

\section{The development of sports industry in the Internet age}

Sports originated from life, and create life, the unique value of sports functions, led people to the pursuit of sports and love for the sports industry laid the foundation for the development prospects. In the traditional field, sports and politics, economy, culture, education, etc., has been in an irreplaceable value. In the era of rapid development of the global Internet economy, the key to the development of the sports industry, the Internet is the key point. Sports and the common development of the Internet is mainly reflected in the intelligent, such as the following aspects:

A. Multi-faceted combination of sports and the Internet

In recent years, the Internet and catering, transportation, film and other industries perfect integration, which will be the integration of the Internet and sports the main trend of development. From the title to the copyright, from the club, star, to the venue, tickets, each link can be extended to deepen.

On the one hand, online ticket purchase will become the mainstream sports tickets, but also have the opportunity and news, event information combined in the same app. On the other hand, sports app can gather a group of high-quality users, and these users of large data, but also have high value. In a comprehensive sports app, users can read the article, watch the video, to achieve payment behavior, etc., can infer a user's interests, habits, travel, economic capacity. The use of large data can predict the outcome of the game, and even race results.

B. The virtualization sports app appears

Appointment venues, vertical areas of the app will gradually appear. On the other hand, for a single event and the development of integrated class app will continue to emerge, such as this year's European Cup and the Olympic Games, have the official app, and review the game time app ranking, these app has a better ranking.

C. Launch of the Internet stadium

The use of the Internet platform, the use of information and communication technology, the Internet and sports venues in the stadium operators, sports venues and other development services to create a new area of ecological. Users can register through the mobile phone APP, through the sports category to find the sports venues you want to view and book, you can also search the stadium by 
location, easy to operate. The progress of science and technology, will eventually be reflected in the sports industry. May change the sports events; Ali, music, as many start-up companies are concerned about the development of VR, and VR technology is mature, through the combination of sports development opportunities to change the concept of sporting experience, to the audience a more intuitive feel .

\section{Summary}

With the rapid advancement of modern science and technology, the extensive use of Internet information, the Internet has been in-depth national life, whether it is education, social production, interpersonal communication, or cultural transmission channels, have reached an irreplaceable role. The use of the Internet will be the main trend to promote the development of China's sports industry. The sports industry itself has huge market potential consumer demand. The sports industry, as a highly correlated industry, can be integrated with other industries, with a strong synergistic effect and Economic benefits.

Sports should break the traditional information service model, the use of the Internet, and constantly tap the potential value of the sports industry, with the Internet platform to promote the development of China's sports industry and innovation, contributed to the development of AAP software, sports activities such as online diversification Development, the future development of the sports industry will be more diversified, diversity, the advantages of the Internet will inject new vitality into the sports industry to promote its gradually mature. The integration of the Internet and sports industry development model to the sports industry has brought new opportunities, so as to stimulate China's economic development of the new points.

\section{References}

[1] Sports Writing Group. Introduction to Sports[M]. Beijing Sport University Press.2013.

[2] XIANG Yu-hong ,YAO Lei.Physical Education Reform in China in th Era of Big Data[j] .Journal of Nanjing Sport Institute .2016.

[3] CHENG Jing-bo.Research on the Reform of the New PE Teaching Mode in the Internet Age[j].Contemporary sports Technology.201608.

[4] ZHANG Seng-mu.Research on Internet + Sports Industry Development Strategy[j].Sports Culture Guide.201603.

[5] HE Gai.The Rise and Development Strategy of "Internet + Sports" Industry[j].Sports Culture Guide.201605. 